\title{
Hybrid Imaging to Assess the Impact of Vulnerable Plaque on Post Myocardial Infarction Myocardial Scar
}

\author{
Alexandra Stănescu1,2,3, Rareș Manuca22, Rodica Togănel1,2, Daniel Cernica1,2, Theodora \\ Benedek1,2,3, Imre Benedek 1,2,3 \\ 1 "George Emil Palade” University of Medicine, Pharmacy, Science and Technology, Târgu Mureș, Romania \\ 2 Clinic of Cardiology, County Clinical Emergency Hospital, Târgu Mureș, Romania \\ ${ }^{3}$ Center of Advanced Research in Multimodality Cardiac Imaging, Cardio Med Medical Center, Târgu Mureș, Romania
}

\section{CORRESPONDENCE}

\section{Rareș Manuca}

Str. Gheorghe Marinescu nr. 50

540136 Târgu Mureș, Romania

Tel: +40 265372653100

E-mail: raresmanuca@gmail.com

\section{ARTICLE HISTORY}

Received: March 5, 2021

Accepted: March 28, 2021
Alexandra Stănescu • Str. 22 Decembrie 1989 nr. 76 540124 Târgu Mureș, Romania. Tel: +40 265217333 , E-mail: alexandrastanescu90@gmail.com

Rodica Togănel • Str. Gheorghe Marinescu nr. 38 , 540139 Târgu Mureș, Romania. Tel: +40 265215551 E-mail: rodicatoganel@yahoo.com

Daniel Cernica • Str. Gheorghe Marinescu nr. 38 540139 Târgu Mureș, Romania. Tel: +40 265215551 Email: daniel.cernica@gmail.com

Theodora Benedek • Str. Gheorghe Marinescu nr. 38 540139 Târgu Mureș, Romania. Tel: +40 265215 551, E-mail: theodora.benedek@gmail.com

Imre Benedek • Str. Gheorghe Marinescu nr. 38 , 540139 Târgu Mureș, Romania. Tel: +40 265215 551, E-mail: imrebenedek@yahoo.com

\begin{abstract}
Background: Multimodality imaging improves the accuracy of cardiac assessment in patients with prior myocardial infarction. The aim of this study was to investigate the association between coronary plaque vulnerability (PV) and myocardial viability in the territory irrigated by the infarctrelated artery (IRA). Secondary objectives include evaluation of the systemic inflammation but also different cardiac risk scores (SYNTAX score, Duke jeopardy score, or calcium score) using hybrid imaging models of coronary computed tomography angiography (CCTA) and cardiac magnetic resonance (CMR) in patients who have suffered a previous myocardial infarction (MI). Material and methods: The study included 45 subjects with documented $\mathrm{Ml}$ in the 30 days prior to study enrolment, who underwent CCTA and CMR examinations. Computational postprocessing of CCTA and CMR images was used to generate fused imaging models. Based on the vulnerability degree of the associated non-culprit lesion located proximally in the IRA, the study population was divided into 3 groups: Group 1 - subjects with no sign of vulnerability ( $n=$ 7); Group 2 - subjects with 1 or 2 CT vulnerability features ( $n=28)$; and Group 3 - subjects with $>2$ features of vulnerability $(n=12)$. Results: CCTA features indicative for the severity of coronary artery disease were not different between groups in terms of calcium scoring ( $460 \pm 501$ vs. 579 \pm 430 vs. $432 \pm 494, p=0.7$ ) or SYNTAX score ( $25 \pm 9.2$ vs. $24.9 \pm 8.3$ vs. $20.2 \pm 11.9, p=0.4)$. However, after 1 month, infarct size and the Duke jeopardy score were associated with increased PV (infarct size $8.77 \pm 3.4 \mathrm{~g}$ in Group 1, compared to $20.87 \pm 8.3 \mathrm{~g}$ in Group 2 and $27.99 \pm 11.8 \mathrm{~g}$ in Group 3 ( $p=0.007$ ), while the Duke jeopardy score was $4.4 \pm 1.6$ in Group 1, vs. $7.07 \pm 2.1$ in Group 2 vs. $7.5 \pm 1.73$ in Group 3 ( $p=0.01$ ). Inflammatory biomarkers were directly associated with coronary plaque vulnerability ( $p=0.007$ for hs-CRP and $p=0.038$ for MMP-9). Conclusion: In patients with prior myocardial infarction, the size of myocardial scar was directly correlated with the vulnerability degree of coronary plaques and with systemic inflammation quantified during the acute phase of the coronary event. Hybrid imaging may help to identify the hemodynamically significant plaques with superior accuracy.
\end{abstract}

Keywords: myocardial infarction, CCTA, MRI, hybrid images, Duke jeopardy score, subtended myocardium, viability 


\section{INTRODUCTION}

It is well known that cardiovascular diseases have the highest mortality rate worldwide. ${ }^{1}$ Innovation in cardiac imaging, mainly based on the cooperation between informatics, cardiology, and radiology, provides learning protocols that improve diagnostic accuracy and offers a wider view on the pathogenesis of different cardiovascular diseases. The concept of hybrid imaging combines more than one imaging methods and offers precious information regarding anatomy and functionality in a spatial view. ${ }^{2}$ Multimodality imaging for the assessment of coronary artery disease (CAD), especially myocardial infarction (MI), has become essential for a complex assessment of these patients. ${ }^{3}$ Coronary computed tomography angiography (CCTA) is a trustworthy method for the assessment of coronary anatomy and severity of CAD, this noninvasive tool becoming more available in many cardiac centers.

CCTA allows 3D cardiac reconstruction, evaluation of coronary plaques, and also a detailed view on the cardiac geometry with high specificity. ${ }^{4-9}$ On the other hand, cardiac magnetic resonance (CMR) is a non-harmful, noninvasive diagnostic tool that provides information regarding functionality, especially kinetics, perfusion, mass, and volume. ${ }^{10-13}$ The integration of information from two different imaging tools allows the combination of the provided parameters to obtain a superior analysis of cardiac function and anatomy, but also to have a better view on patient outcomes and risk stratification.

In order to evaluate the impact of a coronary artery stenosis on myocardial function and structure, many scientists have developed different scores that were difficult to apply in clinical practice sometimes, but their effectiveness on risk prediction has been proven on patients with known CAD or with history of acute myocardial infarction (AMI), including the SYNTAX Score, coronary calcium score, or a more complex assessment - the residual SYNTAX score. However, in the age of advanced technology and artificial intelligence, there are machine learning algorithms that can automatically evaluate the myocardial mass subtended by a coronary lesion. ${ }^{14}$ Also, there are relatively simple methods, such as calculating the Duke jeopardy score, in order to estimate the percentage of affected myocardium in ischemic heart disease. ${ }^{15}$ This cardiac score is used for calculating the percentage of myocardial tissue at risk, based on the location of a coronary artery stenosis. ${ }^{16}$ For calculating this score, the coronary artery tree is divided into 6 segments, and each segment presenting stenosis of more than $75 \%$ is attributed 2 points. Therefore, the maximum Duke jeopardy score can be 12 points. ${ }^{15}$ Investigation of the impact of CAD on myocardial function and structure may provide details regarding myocardial viability. This is compulsory for evaluating the ability of recovering an ischemic myocardial territory through coronary revascularization. It is well known that the gold standard method for appraisal of myocardial viability is fluorodeoxyglucose positron emission tomography (F-FDG PET). ${ }^{17-20}$ Due to high costs and radiation exposure, which may range from 7 to $9 \mathrm{mSV}$, its availability is still low. This imaging procedure allows the investigation of cardiac function, while simultaneously providing information regarding myocardial metabolism and perfusion..$^{21}$ Nevertheless, CMR evaluation, especially using late gadolinium enhancement sequences (LGE), allows the differentiation of viable from non-viable myocardium, in contrast to nuclear scintigraphy which allows only indirectly evaluation of the viable myocardium..$^{22}$ Viability evaluation prior to revascularization of stenotic coronary lesions is advantageous for guiding the right treatment, and mostly to predict functional recovery after blood flow restauration through coronary revascularization. From this point of view, it is also important to mention the animal study conducted by Force $e t a l$., which developed the concept of tethering myocardium, i.e. the viable myocardium juxtaposed to cicatricial regions which might not heal after proper blood flow restaurantion. ${ }^{23}$

\section{OBJECTIVES}

The main objective of the study was to generate fused models based on hybrid CCTA/CMR imaging for the complex evaluation of myocardial viability and vulnerable coronary plaques after an acute myocardial infarction, and to investigate its reliability in assessing the association between non-culprit plaque vulnerability and myocardial function in the territory irrigated by the coronary artery presenting with vulnerable plaques. Secondary, we aimed to investigate a possible relation between systemic inflammation and the amount of myocardial fibrosis reflected by infarct size, mass, and percentage in patients with vulnerable coronary plaques.

\section{MATERIAL AND METHODS}

\section{Study Population}

This original research was a prospective observational study conducted in the Laboratory of Advanced Research in Cardiac Multimodal Imaging of the Cardio Med Medical Center in Târgu Mureş, Romania. We included 45 subjects with documented MI 30 days prior to study enrollment. 
All subjects gave signed consent, and all study procedures were approved by the ethics committee of the institution and were in line with the principles stipulated in the Declaration of Helsinki.

We excluded subjects with any malignancy, renal diseases with abnormal levels of creatinine or known allergy to iodine contrast, as well as pregnant patients. Venous blood samples were collected in order to determine the level of inflammatory biomarkers at day 1 following the acute event: highly-sensitive $\mathrm{C}$ reactive protein (hs-CRP) and matrix metalloproteinase 9 (MMP-9), and also complete blood count and CK-MB levels. During initial hospitalization for infarction, all subjects underwent invasive coronary angiography and cardiac ultrasound. At one month after the acute event, all patients underwent CCTA and CMR assessment.

\section{CCTA acquisition protocol and image post-processing}

All CCTA examinations were performed using a Siemens Somatom Definition 128-slice scanner (Siemens Healthcare GmbH, Erlangen, Germany). All CCTA images were examined by two experienced investigators.

Prior to examination, the heart rate was recorded in all subjects, and those who had a heart rate higher than 65 bpm received beta-blockers in order to decrease the heart rate to $60 \mathrm{bpm}$.

The first image acquisition was performed in a frontal plane topogram, followed by a native acquisition and dynamic contrast administration, using venous right antecubital approach and an automatic syringe, with a rate of contrast administration of $5 \mathrm{~mL} / \mathrm{s}$, followed by $50 \mathrm{~mL}$ saline flush administration.

The following CCTA features of plaque vulnerability were assessed: positive remodeling (PR), spotty calcifications (SC), low-attenuation plaque (LAP), and napkin-ring sign (NRS).23-28 All coronary plaques were evaluated by two experienced radiologists, and for each plaque we calculated a vulnerability score based on CCTA markers. The presence of each marker was assigned 1 point. We considered only vulnerable plaques located upstream of the culprit lesion that irrigated the infarcted subtended myocardium. Based on this vulnerability score, the study population was divided into three groups: Group 1, consisting of 7 patients without vulnerable plaques; Group 2, consisting of 28 patients with moderate plaque vulnerability ( 1 or 2 vulnerability markers); and Group 3, consisting of 12 patients with high vulnerability degree plaques (more than 2 CCTA-based vulnerability markers). We calculated the
Duke jeopardy score, the SYNTAX score, and the calcium score based on CCTA image acquisition in all patients.

\section{CMR acquisition protocol}

In all patients, the applied CMR acquisition protocol focused on the delayed enhancement sequences and used a 1.5T Siemens Magnetom Aera equipment. All myocardial segments were examined during late gadolinium enhancement, and all CMR images were reviewed by two experienced radiologists, who assessed the presence and extension of ischemic lesions, possible edema in $\mathrm{T} 2$ sequences, ejection fraction, myocardial mass, volumes, and contractility.

All subjects underwent invasive coronary angiography during the acute coronary event using a Siemens biplane system, and coronary lesions located contiguous to the infarcted area were mapped by retrospective analysis of the recorded examinations.

\section{Computational CCTA/CMR image post-processing}

Computational CT post-processing was performed using Siemens SyngoVia software (Siemens Medical Solutions, Erlangen, Germany). The 3D reconstruction of the coronary arteries was performed, and coronary arteries were individually reconstructed around their axis.

Advanced analysis of coronary plaques was performed using a semi-automatic image algorithm which detects the vascular contour, on the same research software and using the platform dedicated to coronary analysis (SyngoVia Frontier Coronary Plaque Analysis, Siemens, Erlangen, Germany).

The CMR images were processed using a dedicated MR software for the quantification of myocardial fibrosis (Q-mass, Medis Suite MR, Leiden, the Netherlands). In order to calculate the myocardium mass and infarct size volume, the transmural extent of the infarction was calculated by manually drawing the endocardial and epicardial contours in each slice of the late enhancement series. Then, the software calculated the myocardium mass automatically, differentiating the healthy myocardium from the non-viable one.

The CCTA image was opened in MM Reading mode with an established research software (SyngoVia Frontier, Siemens). The Coronary 0.6 slices were selected, and then a CMR image of the same subject was added. The software was designed to recognize if the imaging method belongs to the same subject, using anatomical particularities. From the CMR images, late enhancement sequences were chosen and then fused with CCTA images, thus obtaining a 


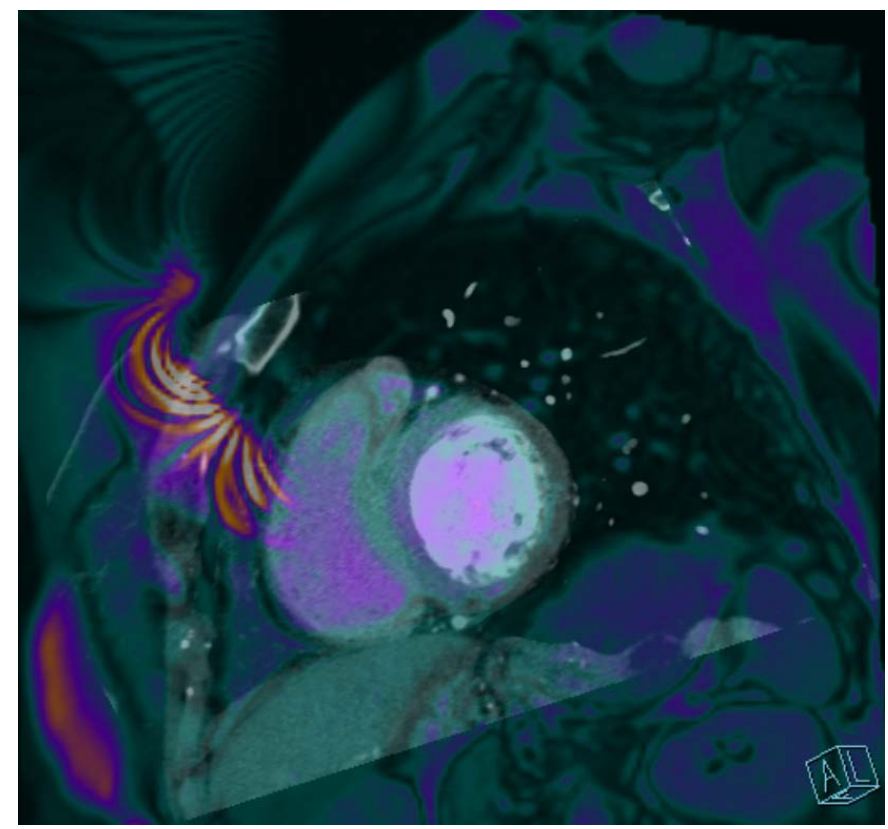

FIGURE 1. Hybrid CCTA/CMR image with CMR reconstruction superposed on the CT image, revealing antero-lateral myocardial ischemia with myocardial scar

hybrid cardiac CCTA \CMR image, as the one exemplified in Figure 1.

\section{Data analysis}

All data were recorded, archived in a dedicated database, and analyzed using Graph Pad Prism 8 software. ANOVA test was used for comparison of quantitative variables be-

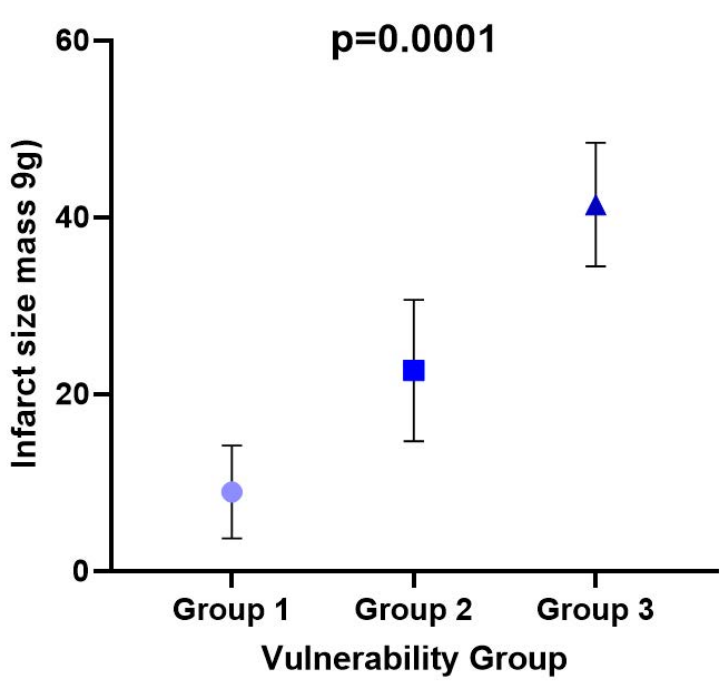

FIGURE 2. Infarct size by CMR and degree of vulnerability by CCTA. The infarct size mass was significantly higher in patients with the highest vulnerability degree of the non-culprit plaque located on the infarct-related artery. tween groups, and the Chi square test and its variants were used to evaluate the association between groups in regard to qualitative data. A p value of 0.05 was considered statistically significant.

\section{RESULTS}

In total, 45 patients were included in the study, with a mean age of 60 years (ranging from 41 to 86 years), $77 \%$ of them being males. General characteristics of the included patients are listed in Table 1. From the total population of the study, $93.3 \%$ suffered from hypertension, $28.8 \%$ had type 2 diabetes mellitus, $40 \%$ were active smokers, and $37.7 \%$ were diagnosed with dyslipidemia. Location of the AMI was in the inferior territory in $46.6 \%$ of the subjects, and $55.5 \%$ had multivessel CAD. There were no statistically significant differences between the three groups regarding left ventricular ejection fraction ( $p=0.5)$, the presence of diabetes mellitus $(p=0.8)$, or tobacco use $(p=0.6)$.

The amount of myocardial necrosis at presentation in the emergency department was not significantly different between the groups, as highlighted by similar levels of CK-MB: $1.153 \pm 1.868 \mathrm{IU} / \mathrm{L}$ in Group 1, $498.3 \pm 428.8 \mathrm{IU} / \mathrm{L}$ in Group 2 , and $2.131 \pm 1.832 \mathrm{IU} / \mathrm{L}$ in Group $3(\mathrm{p}=0.08)$. However, after 1 month, the infarct size and the Duke jeopardy score were both significantly higher in patients with vulnerable plaques. Infarct size at one month was $8.77 \pm 3.4 \mathrm{~g}$ in Group 1, compared to $20.87 \pm 8.3 \mathrm{~g}$ in Group 2 and $27.99 \pm 11.8 \mathrm{~g}$ in Group 3 ( $\mathrm{p}=0.007$ ), as shown in Figure 2, while the Duke jeopardy score was $4.4 \pm 1.6$ in Group 1, $7.07 \pm 2.1$ in Group 2 and $7.5 \pm 1.73$ in Group 3 ( $p=0.01$ ) (Figure 3 ).

Inflammatory biomarkers were directly associated with non-culprit coronary plaque vulnerability (Figure 4). Hs-

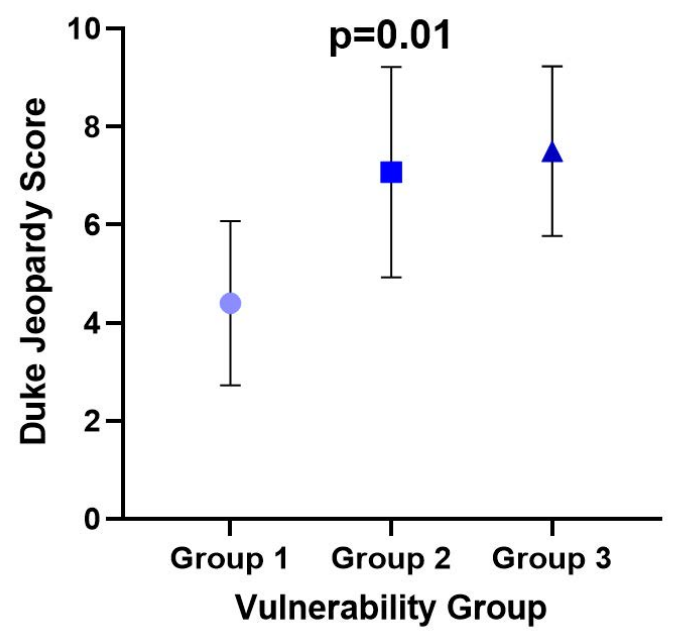

FIGURE 3. The Duke jeopardy score and degree of vulnerability 

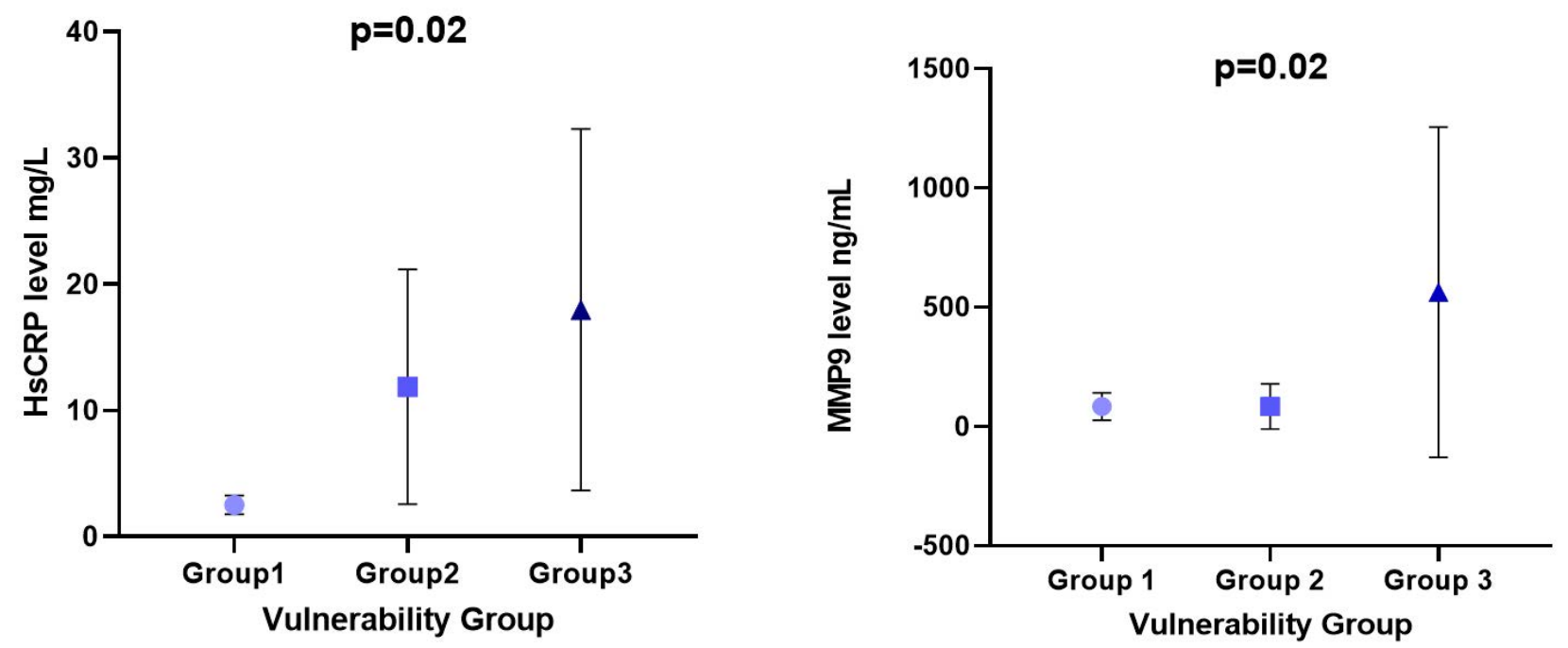

FIGURE 4. Systemic biomarkers associated with increased inflammation (hsCRP and MMP-9) and plaque vulnerability. The highest serum levels of inflammatory biomarkers were observed in patients with the highest plaque vulnerability degree.

CRP was $2.5 \pm 0.7 \mathrm{mg} / \mathrm{L}$ in Group 1 vs. $11.86 \pm 9.3 \mathrm{mg} / \mathrm{L}$ in Group 2 and $17.98 \pm 14.31 \mathrm{mg} / \mathrm{L}$ in Group $3(\mathrm{p}=0.02)$. MMP-9 was significantly higher in patients with advanced vulnerability of the coronary plaques $(563.2 \pm 691.6 \mathrm{ng} /$ $\mathrm{mL}$ in Group 3 vs. $83.71 \pm 94.47 \mathrm{ng} / \mathrm{mL}$ in Group 2 and 84.4 $\pm 57.56 \mathrm{ng} / \mathrm{mL}$ in Group 1, $\mathrm{p}=0.02$ ) (Table 2).

Interestingly, CCTA characteristics associated with the severity of CAD were not significantly different between groups in terms of calcium score (460 \pm 501 vs. $579 \pm 430$ vs. $432 \pm 494, \mathrm{p}=0.7)$ or SYNTAX score ( $25 \pm 9.2$ vs. 24.9 \pm 8.3 vs. $20.2 \pm 11.9, \mathrm{p}=0.4)($ Figure 5$)$.

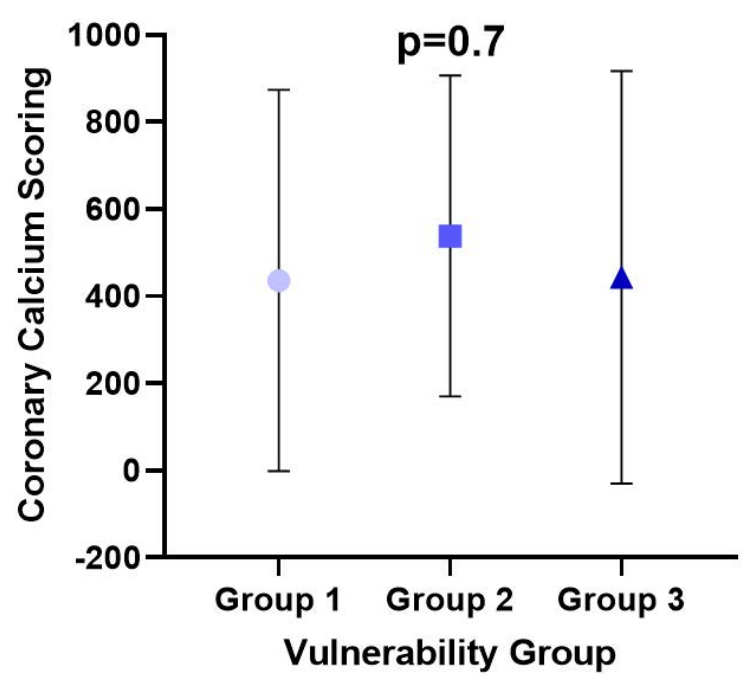

\section{DISCUSSIONS}

This manuscript is part of the FUSE-HEART study, a complex research based on imaging markers, which aims to provide more information regarding myocardial viability, coronary plaques, and inflammation in patients who have suffered an AMI. The present study aimed to investigate possible correlations between inflammation status, myocardial viability assessed by $\mathrm{CMR}$, and coronary plaque vulnerability of non-culprit lesions located on the infarct related artery, as assessed by CCTA.

FIGURE 5. CT markers of atherosclerosis severity in the study population: coronary calcium score and SYNTAX score - comparative analysis between the three plaque vulnerability groups 
TABLE 1. Clinical and echocardiographic characteristics in the population of the study. All values are expressed as mean (SD).

\begin{tabular}{lccc}
\hline & Group 1 & Group 2 & Group 3 \\
\hline Age, years & $61(7.35)$ & $62.14(10.72)$ & $58.08(10.18)$ \\
Weight, kg & $85(15.3)$ & $84.50(15.18)$ & $79.50(8.90)$ \\
Leukocyte no., cells/uL & $8001(1923)$ & $8066(1682)$ & $9288(1961)$ \\
Ejection fraction, \% & $48(2.19)$ & $46.41(4.77)$ & $47(4.56)$ \\
End-diastolic left ventricle diameter, mm & $50.8(4.15)$ & $52.92(5.36)$ & $54.56(4.48)$ \\
\hline
\end{tabular}

The concept of vulnerable patients and vulnerable coronary plaques was first described several years ago. In 1989, Muller et al. used the terminology of vulnerable plaques for the first time, to identify atherosclerotic coronary plaques responsible for most cardiovascular sudden events due to rupture. ${ }^{24,25}$ In this regard, in 2003, Morteza et al. provided a more detailed definition of the vulnerable plaque: a plaque that has a high probability of rupture, with subsequent thrombosis, one that can become a culprit lesion in the near future. ${ }^{26}$ Most of the major criteria that define this type of plaque are based on histological and intravascular imaging studies, which indicate the presence of active inflammation expressed by monocytes, macrophage and Tcell infiltration, or the aspect of plaque with a thin cap with rich lipid core. ${ }^{27,28}$ There are also noninvasive characteristics of vulnerable coronary plaques that can be evaluated using CCTA. These features include positive remodeling, spotty calcifications, low attenuation, and the napkinring sign. ${ }^{29-34}$ However, cardiovascular risk stratification involves more than the presence of vulnerable coronary plaques; it also includes the concepts of vulnerable blood and vulnerable myocardium. Together with documented systemic inflammation, all these meet the criteria to define the vulnerable patient, who presents a high probability to develop an acute coronary syndrome or sudden cardiac death. ${ }^{26}$
Many imaging procedures have improved in the last decades, in order to achieve a better diagnostic accuracy and for evaluating the vulnerable patient in order to predict the risk of major adverse cardiovascular events (MACE).

Our study aimed to demonstrate how inflammation and myocardial fibrosis influence the coronary plaques located in proximity of culprit lesions that have already caused an AMI. The identified associations have highlighted that even in the absence of any statistically significant difference between the groups in terms of myocardial necrosis, systemic inflammatory biomarkers (MMP-9 or hs-CRP) were significantly higher in the group of subjects with a higher vulnerability degree of non-culprit coronary plaques located in the infarct-related artery. This association shows the detrimental effect of inflammation on coronary plaque vulnerability. Moreover, we found that the infarct size mass expressed in grams was more increased in the group with the highest degree of plaque vulnerability, which suggests that pancoronary vulnerability may influence myocardial healing following an acute coronary event, after proper revascularization.

According to our knowledge, this is the first study to use hybrid imaging and fused reconstructions of CCTA and CMR images to demonstrate the correlation between infarct size and non-culprit plaque vulnerability. First, we developed a protocol for obtaining fused images, then we

TABLE 2. Multimodality imaging characteristics and systemic biomarkers in the study groups. All values are expressed as mean (SD)

\begin{tabular}{lcccc}
\hline & Group 1 & Group 2 & Group 3 & p value \\
\hline Infarct size mass, g & $8.94(5.25)$ & $22.70(7.9)$ & $41.45(6.9)$ & 0.0001 \\
SYNTAX score & $25.40(8.042)$ & $25.18(8.4)$ & $21.08(11.32)$ & 0.4226 \\
Ca score, HU & $436(437.5)$ & $538.3(368.2)$ & $443.8(473.5)$ & 0.74 \\
Hs-CRP, mg/L & $2.52(0.74)$ & $9.12(6.19)$ & $16.36(11.73)$ & 0.007 \\
Duke jeopardy score & $4.4(1.67)$ & $7.07(2.14)$ & $7.5(1.73)$ & 0.01 \\
CK-MB, IU/L & $1153(1868)$ & $498.3(428.8)$ & $2131(1832)$ & 0.08 \\
MMP-9, ng/mL & $130(70)$ & $101.3(52)$ & $477(329)$ & 0.038 \\
\hline
\end{tabular}


tested the prototype in clinical settings, on 45 patients with prior MI. This is the first study of its kind which has successfully demonstrated the role of advanced multimodality imaging in the assessment of patients with CAD and vulnerable coronary plaques.

\section{CONCLUSIONS}

In patients with prior myocardial infarction, the size of myocardial scar is directly correlated with the vulnerability degree of non-culprit coronary plaques located on the infarct-related artery and with systemic inflammation evaluated during the acute event. Hybrid imaging may help to identify hemodynamically significant vulnerable plaques with superior accuracy. The integration of hybrid imaging with inflammatory biomarkers may improve risk stratification for major adverse cardiovascular events in the postinfarction period.

\section{CONFLICT OF INTEREST}

Nothing to disclose.

\section{ACKNOWLEDGEMENT}

This research was supported via the research grant no. 103544/2016 - PLaqueIMAGE, contract number 26/01.09.2016, financed by the Romanian Ministry of European Funds, the Romanian Government and the European Union.

\section{REFERENCES}

1. WHO. The global burden of disease: 2004 update. Geneva: World Health Organization; 2008b. https://www.who.int/healthinfo/global_burden_ disease/2004_report_update/en/

2. Saeed M, Van TA, Krug R, Hetts SW, Wilson MW. Cardiac MR imaging: current status and future direction. Cardiovasc Diagn Ther. 2015;5:290310.

3. Stănescu A, Opincariu D, Rat N, et al. Hybrid Imaging in the Assessment of Myocardial Ischemia and Viability. Journal of Interdisciplinary Medicine. 2016:3:242-246

4. Danad I, Raijmakers PG, Appelman YE, et al. Hybrid imaging using quantitative H2 150 PET and CT-based coronary angiography for the detection of coronary artery disease. J Nucl Med. 2013;54:55-63.

5. Partington SL, Kwong RY Dorbala S. Multimodality imaging in the assessment of myocardial viability. S Heart Fail Rev. 2011;16:381-395.

6. Kajander S, Joutsiniemi E, Saraste M, et al. Cardiac positron emission tomography/computed tomography imaging accurately detects anatomically and functionally significant coronary artery disease. Circulation. 2010;122:603-613.

7. Rispler S, Keidar Z, Ghersin E, et al. Integrated single-photon emission computed tomography and computed tomography coronary angiography for the assessment of hemodynamically significant coronary artery lesions. J Am Coll Cardiol. 2007:49:1059-1067.

8. Mowatt G, Cummins E, Waugh N, et al. Systematic review of the clinical effectiveness and cost-effectiveness of 64-slice or higher computed tomography angiography as an alternative to invasive coronary angiography in the investigation of coronary artery disease. Health Technol Assess. 2008;12:iii-iv, ix-143.

9. Masuda A, Yamaki T, Kunii H, et al. Simultaneous Assessment of Myocardial Viability With 18F-fluorodeoxyglucose Uptake and Late Gadolinium Enhancement by PET/MRI. Circulation. 2016;134:A11929.

10. Leone AM, De Caterina AR, Basile E, et al. Influence of the amount of myocardium subtended by a stenosis on fractional flow reserve. Circ Cardiovasc Interv. 2013;6:29-36

11. Selvanayagam JB, Kardos A, Francis JM, et al. Value of delayedenhancement cardiovascular magnetic resonance imaging in predicting myocardial viability after surgical revascularization. Circulation. 2004:110:1535-1541.

12. Allman KC, Shaw LJ, Hachamovitch R, Udelson JE. Myocardial viability testing and impact of revascularization on prognosis in patients with coronary artery disease and left ventricular dysfunction: a meta-analysis. $J$ Am Coll Cardiol. 2002;39:1151-1158

13. Thiele H, Kappl MJ, Conradi S, Niebauer J, Hambrecht, Schuler G. Reproducibility of chronic and acute infarct size measurement by delayed enhancement-magnetic resonance imaging. J Am Coll Cardiol. 2006:47:1641-1645

14. Ide S, Sumitsuji S, Yamaguchi O, Sakata Y. Cardiac computed tomographyderived myocardial mass at risk using the Voronoi-based segmentation algorithm: a histological validation study. J Cardiovasc Comput Tomogr. 2017:11:179-182

15. Califf RM, Phillips HR, Hindman MC, et al. Prognostic value of a coronary artery jeopardy score. J Am Coll Cardiol. 1985:5:1055-1063.

16. Dash H, Johnson RA, Dinsmore RE, Hawthorne JW. Cardiomyopathic syndrome due to coronary artery disease. I. Relation to angiographic extent of coronary artery disease and to remote myocardial infarction. $\mathrm{Br}$ Heart J. 1977:39:733-739.

17. Mahrholdt H, Wagner A, Judd R.M, Sechtem U, Kim RJ. Delayed enhancement cardiovascular magnetic resonance assessment of nonischaemic cardiomyopathies. Eur Heart J. 2005;26:1461-1474.

18. Wijns W, Vatner SF, Camici PG. Hibernating myocardium. N Engl J Med 1998:339:173-181

19. Tillisch J, Brunken R, Marshall R, et al. Reversibility of cardial wallmotion abnormalities predicted by positron tomography. N Engl J Med. 1986;314:884-888.

20. Maddahi J Schelbert H, Brunken R, Di Carli M. Role of thallium-201 and PET imaging in evaluation of myocardial viability and management of patients with coronary artery disease and left ventricular dysfunction. $J$ Nucl Med. 1994;35:707-715

21. Christopher J. PET vs. MRI for Myocardial Viability. Indian Journal of Clinical Cardiology. 2020;1:40-45.

22. Kim SY, Kim KS, Seung MJ, et al. The culprit lesion score on multi-detector computed tomography can detect vulnerable coronary artery plaque. Int J Cardiovasc Imaging. 2010;26:245-252.

23. Force $T$, Kemper A, Perkins L, et al. Overestimation of infarct size by quantitative two-dimensional echocardiography: the role of tethering and of analytic procedures. Circulation. 1986;73:1360-1368.

24. Muller J, Tofler G, Stone P. Circadian variation and triggers of onset of acute cardiovascular disease. Circulation. 1989:79:733-743.

25. Muller JE, Abela GS, Nesto RW, et al. Triggers, acute risk factors and vulnerable plaques: the lexicon of a new frontier. J Am Coll Cardiol. 1994:23:809-813

26. Naghavi M, Libby $\mathrm{P}$, Falk E, et al. From vulnerable plaque to vulnerable patient: a call for new definitions and risk assessment strategies: Part I. Circulation. 2003:108:1664-1672.

27. Obaid DR, Calvert PA, Gopalan D, et al. Atherosclerotic plaque composition and classification identified by coronary computed tomography: assessment of computed tomography-generated plaque maps compared with virtual histology intravascular ultrasound and histology. Circ Cardiovasc Imaging. 2013;6:655-664.

28. Pundziute G, Schuijf JD, Jukema JW, et al. Head-to-head comparison of coronary plaque evaluation between multislice computed tomography and intravascular ultrasound radiofrequency data analysis. J Am Coll Cardiol Intv. 2008:1:176-182.

29. Motoyama S, Kondo T, Sarai M, et al. Multislice computed tomographic characteristics of coronary lesions in acute coronary syndromes. J Am Coll Cardiol. 2007;50:319-326

30. Marwan M, Taher MA, El Meniawy K, et al. In vivo CT detection of lipidrich coronary artery atherosclerotic plaques using quantitative histogram analysis: a head to head comparison with IVUS. Atherosclerosis 2011:215:110-115 
31. Maurovich-Horvat P, Hoffmann $U$, Vorpahl M, et al. The napkin-ring sign: CT signature of high-risk coronary plaques? JACC Cardiovasc Imaging. 2010;3:440-444.

32. Maurovich-Horvat P, Ferencik M, Voros S, et al. Comprehensive plaque assessment by coronary CT angiography. Nat Rev Cardiol. 2014;11:390402.

33. Feuchtner $G$, Kerber J, Burghard $P$, et al. The high-risk criteria lowattenuation plaque $<60 \mathrm{HU}$ and the napkin-ring sign are the most powerful predictors of MACE: a long-term follow-up study. Eur Heart J Cardiovasc Imaging. 2017;18:772-779.

34. Hoffmann U, Moselewski F, Nieman K, et al. Noninvasive assessment of plaque morphology and composition in culprit and stable lesions in acute coronary syndrome and stable lesions in stable angina by multidetector computed tomography. J Am Coll Cardiol. 2006;47:1655-1662. 